\title{
BULLE'TIN OF THE
}

\section{AMERICAN MATHEMATICAL SOCIETY}

\section{'THE SUMMER MEETING OF THE AMERICAN MATHEMATICAL SOCIETY.}

The first summer meeting of the American Mathematical Society was held in one of the lecture-rooms of the Polytechnic Institute in Brooklyn, N. Y., on 'Tuesday and Wednesday, August 14 and 15, 1894 . These days immediately preceded those fixed for the meeting of the American Association for the Advancement of Science, and all concessions obtained by that Association from railways and hotels were extended to the members of the Mathematical Society. 'The members of the Society were invited to participate also in the entertainments and excursions arranged in honor of the Association. To the co-operation of that larger organization thus was due in part the success of the Society's meeting. Among those present were Dr. E. M. Blake, Professor Henry B. Fine, Professor Thomas S. Fiske, Professor G. B. Halsted, Dr. George W. Hill, Professor E. W. Hyde, Mr. P. A. Lambert, Dr. Emory McClintock, Professor Alexander Macfarlane, Professor Mansfield Merriman, Professor E. Hastings Moore, Professor Frank Morley, Professor Hubert A. Newton, Professor George D. Olds, Mr. J. C. Pfister, Mr. Ralph A. Roberts, Professor James B. Shaw, Jr., Mr. J. F. Shields, Professor Ormond Stone, Dr. Edward B. Van Vleck, Professor L. G. Weld, Professor R. S. Woodward, and Professor Alexander Ziwet. Althongh the attendance was not so large as had been anticipated, the interest was well maintained throughout. On Wednesday evening, after adjournment, a dinner was given at the St. George Hotel, in which a majority of the members who had attended the meeting participated.

The Society elected the following new members: Rev. John G. Hagen, S.J., Georgetown College Observatory, West Washington, D.C. ; Dr. Gustavus Hay, Boston, Mass. ; Professor Hubert A. Newton, Yale University, New Haven, Conn.; Professor Harry Walter Tyler, Massachusetts Institute of Technology, Boston, Mass. 
The following papers were presented :

1. Theorems in the calculus of enlargement. Dr. Emory McClintock, New York, N. X.

2. A method for calculating simultaneously all the roots of an equation. Dr. Emory McClintock, New York, N. Y.

3. Elliptic functions and the Cartesian curve. Professor Frank Morley, Haverford, Pa.

4. Concerning the definition by a system of functional properties of the function $f(z)=\frac{\sin \pi z}{\pi}$. Professor E. Hastings Moore, Chicago, Ill.

5. Bertrand's paradox and the non-euclidean geometry. Professor George Bruce Halsted, Austin, Texas.

6. Analytical theory of the errors of interpolated values from numerical tables. Professor R. S. Woodward, New York, N. Y.

7. Upon the problem of the minimum sum of the distances of a point from given points. Professor V. Schlegel, Hagen, Germany.

8. On the fundamental laws of algebra. Professor Alexander Macfarlane, Austin, Texas.

9. About cube numbers whose sum is a cube number. Dr. Artemas Martin, Washington, D.C.

10. Reduction of the resultant of a binary quadric and $n$-ic by virtue of its semicombinant property. Professor Henry S. White, Evanston, Ill.

In the absence of their authors, paper No. 7 was presented by Professor Hyde, paper No. 9 by the Secretary, and No. 10 by Professor Ziwet.

Nos. 1, 2, 3, are intended for publication in the American Journal of Mathematics; Nos. 4, 6, in the Annals of Mathematics; No. 5 in the Proceedings of the Texas Academy of Science; Nos. 7,10 , in the Bulletin of the American Mathematical Society; No. 9, in the Mathematical Magazine.

In the first of Dr. McClintock's papers the formulæ developed are all special cases of the most general theorem presented, viz. :

$$
f E=f z+f E_{0} 0^{|2|} \cdot H+f E_{0} 0^{\mid 21} \cdot H^{2} / 2 !+\ldots,
$$

where $E$ is an operation such that, applied upon any function, say $\psi x$, we shall have $E^{h} \psi x=\psi(x+h) ; H$ is a function of $E$ depending on some other function $\phi E$ and defined by $H h \phi E=E^{h}-z^{h} ; f E_{0} 0^{|m|}$ means $f E_{x} x^{|m|}{ }_{[x=0]} ;$ and $x^{\mid m !}=$ $x\left(\phi E_{x}\right)^{m} z^{x-m h}(x-h)^{0}(x-2 h) \ldots(x-m h+h)$. Since $\psi E_{0} y^{\circ}=\psi y$, an important case, derived by operating with the chief series upon $y^{\circ}$, is

$$
f y=f z+f E_{0} 0^{|1|} \cdot x+f E_{0} 0^{\mid 21} \cdot x^{2} / 2 !+\ldots,
$$


where $x$ is the same function of $y$ as $H$ of $E$, defined by $x h \phi y=y^{h}-z^{h}$. If, for a special case of this, $h=1$, the result is identical in value with Lagrange's theorem, but with no reference to differentiation in the form of the coefficients.

The second paper contains a remarkable application of the series last quoted to the theory of equations. The general formula, applicable to any equation $x^{n}=c+n \phi x$, is, after putting $\omega^{n}=c$,

$x=\omega+\omega^{1-n} \phi \omega+E_{0}\left(\phi E_{0}\right)^{2} \omega^{0-2 n}(0-n) / 2 !$

$$
+E_{0}\left(\phi E_{0}\right)^{3} \omega^{0-3 n}(0-n)(0-2 n) / 3 !+\ldots
$$

The simplest form of $c$ is 1 or -1 , and when the equation is arranged in that form this series, if convergent, gives all the roots upon substituting for $\omega$ the several $n$th roots of 1 or of -1 , as the case may be. If $c$ is not 1 or -1 , it will be $c=\omega^{n}=\zeta^{n} \xi^{n}$, where $\zeta^{n}$ is the numerical value of $c$, and $\xi^{n}$ is 1 or -1 , and in this case $\omega$ is to be replaced by $\zeta \xi$, $\xi$ having the several $n$ values of the roots of 1 or of -1 . The formula holds when $n$ has any value, positive or negative, though nothing is gained by adjusting the equation with $n$ negative or fractional; and in particular the term chosen for $x^{n}$ need not be the highest power present, though in that case, of course, only $n$ roots will be developed by the series at once. Especial attention is given to the trinomial equation $x^{n}=\omega^{n}+n a x^{n-k}$, wherein $k$ is not necessarily positive. For this equation the general formula becomes

$$
\begin{aligned}
& x=\omega+\omega^{1-k} \cdot a+\omega^{1-2 k}(1-2 k+n) \cdot a^{2} / 2 ! \\
& . \quad+\omega^{1-3 k}(1-3 k+n)(1-3 k+2 n) \cdot a^{3} / 3 !+\ldots
\end{aligned}
$$

This formula, though actually first obtained as here described, may be derived from Lagrange's theorem, by putting $x^{n}=u$, $\omega^{n}=z, \phi x=\phi\left(u^{\frac{1}{n}}\right)$, and expanding $u^{\frac{1}{n}}$. Similarly, the general formula may be used in this alternative form, likewise derived, as an afterthought, from Lagrange's theorem :

$$
\begin{aligned}
x=\omega+\omega^{1-n} \phi \omega+\omega^{1-n} & \frac{d}{d \omega} \omega^{1-n}(\phi \omega)^{2} / 2 ! \\
& \quad+\left(\omega^{1-n} \frac{d}{d \omega}\right)^{2} \omega^{1-n}(\phi \omega)^{3} / 3 !+\ldots .
\end{aligned}
$$

The trinomial formula is convergent whenever $n$ is positive and the numerical value of $a^{n}$ is less than that of

$$
k^{-k}(n-k)^{k-n} \cos ^{n k}
$$

Its use is illustrated by the complete exhibition of the six imaginary roots of $x^{6}+x+1=0$. When $n$ is negative, the trinomial formula gives the same series as if both sides of the equation were multiplied by $x^{-n}, x^{n}= \pm 1+n a x^{n-k}$ 
becoming $x^{-n}= \pm 1 \mp n a x^{-k}$. If the value of $a$ is such that the series for the trinomial $x^{n}= \pm 1+n a x^{n-k}$ is not convergent, it is shown that the series for $x^{n-k}=$ $\left(\mp 1+x^{n}\right) / n a$ must be convergent, yielding $n-k$ of the $n$ roots, and that the other $k$ roots may be obtained either by putting $x=u^{-1}$ and multiplying throughout by $u^{n}$, whence $u^{k}=\left(1 \mp u^{n}\right) / n a$, or by using $x^{k}=n a \pm x^{k-n}$, both forms yielding convergent series. When the reciprocal transformation $x=u^{-1}$ is employed, it is convenient to use one of two formulæ; * viz., for the trinomial $u^{n}=\omega^{n}+n a u^{n-k}$,

$x=u^{-1}=\omega^{-1}-\omega^{-1-k} \cdot a-\omega^{-1-2 k}(-1-2 k+n) \cdot a^{2} / 2 !$

$-\omega^{-1-3 k}(-1-3 k+n)(-1-3 k+2 n) \cdot a^{3} / 3 !-\ldots$,

and for the general equation $u^{n}=\omega^{n}+n \phi u$,

$x=u^{-1}=\omega^{-1}-\omega^{-1-n} \phi \omega-E_{0}^{-1}\left(\phi E_{0}\right)^{2} \omega^{0-2 n}(0-n) / 2 !-\ldots$

Professor Morley's paper aims at showing how the properties of the so-called Cartesian oval, both those that are known and others, can be systematically and easily developed by means of Weierstrass's elliptic functions. The following topics are considered : The Cartesian oval as a normal elliptic curve. The relations between distances of a point from the single foci, the triple focus, and the double tangent. Abel's theorem for elliptic functions of the second kind. The intersection of a circle and the curve. The bitangent circles. The circles of curvature. The tangents from a point.

The system of functional properties receiving principal consideration in Professor Moore's paper is the following: (1) $f(z)$ is an integral analytic function of the complex variable $z$. It has as its complete system of zeros, $z=m, m=0, \pm 1$, $\pm 2, \pm 3, \ldots$, the multiplicity of each zero being unity, and limit when $z=0$ of $f(z) / z=1$. (2) $f^{\prime}(2 z) f\left(\frac{1}{2}\right)=2 f^{\prime}(z) f^{\prime}\left(z+\frac{1}{2}\right)$. (3) $f(-z)=-f(z)$. This furnishes a new determination of the external exponential factor $\left(e^{g(z)}=1\right)$ in the expression of the function $f(z)$ as a Weierstrassian infinite product:

$$
f(z)=\frac{\sin \pi z}{\pi}=e^{g(z)} z \Pi_{m}^{\prime}\left(1-\frac{z}{m}\right) e^{\frac{z}{m}} \quad[m \neq 0]
$$

* These reciprocal formulæ have been communicated to the Editors of the Bulletin by Dr. McClintock, having been devised by him shortly after reading the paper before the Society. He has undertaken to read a second paper at the next meeting, in which he will indicate the man ner whereby, by having regard to the larger or " dominant" coefficients, any equation may apparently be so adjusted as to yield one or more convergent series for all the roots, and will illustrate the separation of roots by this method by all of the many examples employed for the application of Sturm's theorem in Burnside \& Panton's Theory of Equations. Equations having imaginary coefficients will also be illustrated, as to which at the reading, in reply to a question, it was said that such equations, though not yet examined, must certainly come within the theory. 
Professor Halsted's paper gives, after a historical introduction, Bertrand's renowned paradox in a new form, and maintains that it is inexplicable withont recourse to the non-euclidean geometry, but with the aid of Lobachersky's theory of the equidistantial is readily shown to involve a petitio principii.

The object of Professor Woodward's paper is to extend and complete the theory of the errors in question. 'The investigation is supplementary to papers already published by the author on this subject in the Analyst, 1882, and in the $A n$ nals of Mathematics, 1886. The special peculiarity of the investigation is that it requires the combination of continuous and discontinuous errors. The quasi-geometrical method followed in the previous papers is now supplanted by a wholly analytical process.

In Professor Schlegel's paper the differential equation between the distances $p_{1}, p_{2}, \ldots p_{n}$ of a variable point $P$ from $n$ fixed points in an $(n-1)$-dimensional space is first derived and the minimum of the function

$$
s=\lambda_{1} p_{1}^{u}+\lambda_{2} p_{2}^{u}+\ldots+\lambda_{n} p_{n}^{u}
$$

of these distances is determined. The general result thus obtained (I) reduces in the case of the plane to an analytical form given by Steiner and furnishes in the case of ordinary space the geometrical condition found by Sturm. To extend the problem to the case of $n$ points in a space of less than $n-1$ dimensions and to gain at the same time a purely geometrical interpretation of the general condition for a minimum, the formula (I) is put into another form (II); then the generalized problem is solved by a formula (III) derived by a different method; this formula (III) is interpreted geometrically and finally shown to reduce to formula (II). Thus the organic connection is established between the general methods of which the results found by Steiner, Sturm, and others are particular cases. Some special cases are next discussed and remarks are made concerning the construction of the minimum point and the formation of the point-system belonging to a given minimum point. The case of a linear row of points is specially investigated and applied to the solution of a practical problem. Finally the results are applied to the problem in mechanics of determining the point of least attraction for a given group of points. The investigation is thronghout carried on by the methods of Grassmann's geometrical calculus.

Professor Macfarlane maintains that the laws of algebra ought to be placed on a real basis, not on a formal basis. The several formal laws are examined, and it is considered whether they are entirely independent. Their validity in space analysis is considered, the exact conditions where some of them break down being pointed out. 
Dr. Martin deduces in his paper a number of formulæ which lead to very remarkable numerical results.

In Professor White's paper the problem is to reduce the resultant of a binary quadric and $n$-ic to a sum of products of invariants of the "reduced system." Clebsch, and later Gordan, have solved it. They use unnecessary auxiliaries. The reduction is accomplished more speedily by constructing synthetically, according to the method of the theory of forms, an expression involving undetermined coefficients whose values are then found by the differential equation of apolarity applied to that covariant which becomes an exact power of the common factor of the two quantities in case their resultant vanishes. Incidentally the connection between apolarity and the semicombinant property is illustrated.

Thomas S. Fiske.

\section{ON THE CONNECTION BETWEEN BINARY QUARTICS AND ELLIPTIC FUNCTIONS.*}

BY PROFESSOR E. STUDY.

AFTER the treatment which the subject has received in the recent work of Professors Harkness and Morley, it may be supposed that American mathematicians are quite familiar with the utilization of the theory of invariants in the theory of elliptic functions. We shall confine ourselves to the simplest case, where the theory of a binary quartic is concerned. We intend to show how a certain group of rational and irrational covariants of a binary quartic can be expressed as one-valued functions of one or two parameters, thus filling up a number of lacunae contained in former presentations of the subject.

After having explained the system of notation we are to apply, we proceed, partly following Cayley, to define a systern of irrational covariants. These being known, we compare these quantics with the elliptic $\theta$-functions; and thus we will be enabled to express the connection in question in very simple terms.

Of course we must suppress here not only the proofs, but also quite a number of details; a full exposition will be published shortly in the American Journal of Mathematics.

\section{Notation.}

Denoting the quartic $f(x)$ symbolically by $f=(a x)^{4}$ $=\left(a_{1} x_{2}-a_{2} x_{1}\right)^{4}$, the following forms constitute, as is well known, what is termed the complete system of $f$ :

* This puper, which Professor Study kindly transmitted for presen tation at the Brooklyn meeting of the Ambrican Mathematical So. CIETY, arrived too late for that purpose. 